\title{
Acetic Acid
}

National Cancer Institute

\section{Source}

National Cancer Institute. Acetic Acid. NCl Thesaurus. Code C61623.

A synthetic carboxylic acid with antibacterial and antifungal properties. Although its mechanism of action is not fully known, undissociated acetic acid may enhance lipid solubility allowing increased fatty acid accumulation on the cell membrane or in other cell wall structures. Acetic acid, as a weak acid, can inhibit carbohydrate metabolism resulting in subsequent death of the organism. 